\title{
EFFECT OF IN-OFFICE BLEACHING TECHNIQUES AND TOPICAL FLUORIDE APPLICATION ON COLOR AND SURFACE ROUGHNESS OF TWO TYPES OF DENTAL CERAMICS (IN-VITRO STUDY)
}

\author{
Fatma M. Soliman*, Lamia E. Dawood** and Shaimaa A. Abo El-Farag**
}

\begin{abstract}
Objective: The purpose of this in-Vitro study was to assess the effect of two different bleaching techniques and topical fluoride application on the color and surface roughness of two types of $\mathrm{CAD} / \mathrm{CAM}$ dental ceramics.

Materials and methods: In this study 40 discs of two types of CAD/CAM dental ceramics were used \{Lithium disilicate glass ceramic (IPS e.max CAD) (Group E) and Zirconia-reinforced lithium silicate ceramic (Vita Suprinity) (Group S) \}. Baseline measurements for color and surface roughness (Ra) were taken using spectrophotometer and Atomic Force Microscope respectively. The specimens of each ceramic material were randomly divided into two subgroups $(\mathrm{n}=10)$ according to the bleaching agent used: a photo-activated Zoom bleaching agent $(\mathrm{Z})$ and a chemically-activated Opalescence Boost $40 \%$ agent (O). Two measurements of color and surface roughness were done: immediately after bleaching application (Immediate I) and after one week from the last bleaching session (Delayed D). Then each specimen from each ceramic material was exposed to Acidulated Phosphate Fluoride (APF) application and final measurements of color and surface roughness values were done. The obtained data were collected, tabulated and statistically analyzed.
\end{abstract}

Results: The results showed that, bleaching had a statistically significant effect on mean color change $\Delta \mathrm{E}$ of the tested ceramic materials in all tested conditions (I, D \&F). No significant difference on mean $\Delta \mathrm{E}$ of the two ceramic materials. The Opalescence Boost $40 \%$ showed higher statistically significant difference in color parameters than the Zoom agent. Vita Suprinity showed higher statistical significant difference in color parameters than E.max CAD. Moreover, ceramic type and bleaching agents had a statistically significant effect on mean Ra. In immediate bleaching, the Vita Suprinity bleached with Zoom gel (SZ) showed the highest mean Ra value while in delayed bleaching and after fluoride treatments, E.max CAD bleached with Opalescence agent (EO) showed the highest mean Ra value. After fluoride treatment, the mean Ra of the tested ceramic materials demonstrated higher values than bleaching only. In addition, there was a positive correlation between color parameters and surface roughness.

\footnotetext{
* Post Graduate Student of Fixed Prosthodontics, Faculty of Dentistry, Mansoura University.

** Associate Professor of Fixed Prosthodontics, Faculty of Dentistry, Mansoura University.
} 
Conclusions: The influence of in-office bleaching agents on color was material dependent. The changes in color and surface roughness were time dependent. Fluoride application following the bleaching of the ceramic materials showed higher color and surface roughness changes than bleaching application only in all tested groups.

Recommendation: The clinician should protect the ceramic restoration before bleaching sessions and/or fluoride application to avoid esthetic deteriorations. The effect of different concentrations and application times could be further being assessed to give better recommendations for the best protocols of bleaching and fluoride agents application that ensures optimum color stability and surface topography characteristics.

KEYWORDS: CAD/CAM dental ceramic, IPS E.max CAD, Vita Suprinity, bleaching agents, ceramics, Zoom, Opalescence, color, surface roughness, esthetics, APF.

\section{INTRODUCTION}

Dental ceramics are used extensively as a restorative material because of their esthetic properties, durability, and biocompatibility. ${ }^{1}$ A smart dentist should know how to satisfy his patients by solving their esthetic problems and fulfilling their esthetic demands using safe and relatively inexpensive materials and methods. Lithium disilicate glass ceramic (IPS e.max CAD) was introduced in 2007 using the CAD/CAM technology. Because of its low refractive index, it was considered very translucent thus offering outstanding esthetic properties, high strength (about $350 \mathrm{MPa}$ ) and can be adhesively bonded. ${ }^{2}$ In 2013, zirconia-reinforced lithium silicate (ZLSs) was introduced, this material comprises a lithium silicate glass ceramic that is strengthened with approximately $10 \%$ zirconia crystals by weight. ${ }^{3}$ The ZLSs offers excellent translucency, fluorescence, strength (flexural strength of 420 $\mathrm{MPa}$ ) and provides excellent esthetic properties. ${ }^{3,4}$

Tooth bleaching is a valuable solution for esthetic problems, in-office and home vital bleaching are widely used because of their effectiveness in removing tooth discoloration and also their conservative approach. ${ }^{5}$ Recently, a broad range of bleaching agents containing different concentrations of carbamide peroxide (CP) and/or hydrogen peroxide (HP) are available. ${ }^{6}$ In-office bleaching technique has emerged because highly concentrated products can promote faster whitening. ${ }^{7} \mathrm{HP}$ acts as a strong oxidizing agent through the formation of free radicals and reactive oxygen molecules which can cause morphologic alterations in the mineralized structures. $^{2}$

The color assessment is influenced by its physical properties, the incident light on the object, the surrounding objects and the subjective assessment of the observer. Spectrophotometers are widely used for color measurements due to their precise measurements, repeatability, and reproducibility. ${ }^{89}$ Surface roughness of a restoration is one of the important factors that determine the long-term success of a restoration..$^{10}$ Roughening of the restoration lead to more plaque accumulation and stain retention which is responsible for gingival inflammation, secondary caries and poor esthetic. ${ }^{1}$ During bleaching procedure, bleaching agent could accidentally touch the dental restorations causing surface roughness. There are several reports on the effect of bleaching agents on properties related to different ceramic surface textures ${ }^{11,14-16}$, one of them ${ }^{12}$ found that, there was a significant increase in surface roughness of feldspathic porcelain surface, while others ${ }^{16,17}$ found no significant alterations. So, there is inadequate information about the effect of bleaching agents on color and surface roughness of ZLSs ceramic.

Some authors ${ }^{13-16}$ have advocated the use of topical fluoride solution to counteract the side effects of bleaching agent on the tooth enamel. Although 
fluoride treatment proved to be beneficial to natural tooth structure, it may have adverse effects on the color and surface texture of dental ceramics. ${ }^{16}$

Therefore, this in-Vitro study was conducted to evaluate the effect of two different bleaching techniques and topical fluoride application on the color as well as the surface roughness of lithium disilicate and zirconia-reinforced lithium silicate ceramics. The null hypothesis was that, in-office bleaching agents and topical fluoride have no effect on the color and surface roughness of the glass ceramic materials.

\section{MATERIALS AND METHODS}

A total number of 40 discs were constructed with standardized dimensions $(10 \mathrm{~mm}$ diameter \& $1.5 \mathrm{~mm}$ thickness). The discs were divided into 2 equal groups $(n=20)$ according to the type of the ceramic material used; either Lithium disilicate glass ceramic; Group E (IPS E.max CAD), or Zirconia re-inforced lithium silicate ceramic; Group S (Vita Suprinity). Each group was further subdivided into two equal subgroups $(\mathrm{n}=10)$ according to the bleaching agent used; either photo-activated bleaching agent Zoom (Z), or chemically-activated bleaching agent Opalescence Boost 40\% (O).

In order to standardize the shape and dimensions of all discs, 3D Autodesk software was used to design the disc virtually. The blocks of the 2 ceramic materials were selected from the milling file in CAD software and mounted in the milling machine to start the milling procedures. The milling procedure, firing cycle, separation of the discs, finishing, polishing and cleaning of the discs were done according to the manufacturer's instructions for each ceramic material. Each disc was stored in labelled test tube containing artificial saliva that was changed daily till the end of measurements. Baseline measurements of color using Spectrophotometer and surface roughness using Atomic Force Microscope (AFM) were done to obtain color parameters and both qualitative and quantitative surface roughness values. Then, the discs were restored inside their numbered test tubes containing the fresh artificial saliva.

Ten specimens from each ceramic group were randomly selected and treated with Zoom agent (EZ\&SZ) for 45 minutes (three sessions), 15 minutes for each session. The other 10 specimens of each group were bleached with Opalescence $40 \%$ (EO\&SO) for 40 minutes (two sessions), 20 minutes for each session. Immediate measurements of color and surface roughness (I) were done to detect the changes occurred immediately after bleaching (EZI, EOI, SZI, SOI) and to obtain the 3D images of surface roughness changes.

After one week from last bleaching session, the specimens were dried and placed inside the chamber of the spectrophotometer first to calculate the delayed color changes then placed inside AFM to calculate the delayed surface roughness values. Delayed measurements (D) were done for all specimen's groups (EZD, EOD, SZD, SOD) and also the 3D images were obtained. Acidulated Phosphate Fluoride (APF) was applied for 1 minute as recommended by the manufacturer on glazed, bleached surface of each specimen. Then, the measurements of color and surface roughness were done after fluoride application (EZF, EOF, SZF, SOF) to assess the effect of fluoride following bleaching on tested ceramic materials and the 3D images were obtained.

All data were collected tabulated and statistically analyzed using SPSS $^{\mathrm{TM}}$ software (Version 23, IBM, USA).

\section{RESULTS}

a) Color change: Regarding the color change $\Delta \mathrm{E}$, there was a statistically significant difference between the ceramic groups (EZ, EO, SZ, SO) in all tested conditions (I, D and F). Regarding each bleaching agent, there was no significant 
difference between the color changes $\Delta \mathrm{E}$ values of both ceramic materials $(\mathrm{E} \& \mathrm{~S})$ in all tested conditions (I, D and F). The SO group showed the highest $\Delta \mathrm{E}$ in all conditions $\mathrm{I}, \mathrm{D}$ and $\mathrm{F}$ $3.51 \pm 0.33,4.51 \pm 0.33 \& 5.06 \pm 0.33$ respectively while the $\mathrm{EZ}$ one showed the least $\Delta \mathrm{E}$ values $2.42 \pm 0.58,3.21 \pm 0.58,3.66 \pm 0.58$ respectively.

b) Surface roughness: The results of surface roughness $(\mathrm{Ra})$ of the two ceramic materials $(\mathrm{E}$ \& S) were shown in Table (1).
The 3D images of tested ceramic materials E \& $\mathrm{S}$ followed by bleaching agents $\mathrm{Z} \& \mathrm{O}$ and topical fluoride treatments are represented in Figures (1\&2).

\section{Correlation between color parameters and (Ra)}

The correlations between color parameters and surface roughness $(\mathrm{Ra})$ for both types of ceramic and bleaching agents are positive (parallel) correlation. The Pearson correlation coefficient between the $\Delta \mathrm{E}$ \& Ra was $0.489 \& 0.124$ for E and S respectively.

TABLE (1): Mean (Ra), standard deviation (SD)values and results of comparison between the four ceramicbleaching mean $(\mathrm{Ra})$ regardless with other variables:

\begin{tabular}{|c|c|c|c|c|c|c|}
\hline \multirow{2}{*}{\multicolumn{2}{|c|}{ Bleaching procedures }} & \multicolumn{4}{|c|}{ Groups } & \multirow{2}{*}{$\begin{array}{c}\text { ANOVA } \\
\text { P-value }\end{array}$} \\
\hline & & \multirow{2}{*}{$\frac{\mathrm{EZ}}{10.09}$} & \multirow{2}{*}{$\begin{array}{c}\text { EO } \\
9.89\end{array}$} & \multirow{2}{*}{$\frac{\mathrm{SZ}}{16.13}$} & \multirow{2}{*}{$\frac{\mathrm{SO}}{12.61}$} & \\
\hline \multirow{2}{*}{ Baseline } & Mean & & & & & \multirow{4}{*}{$0.002 *$} \\
\hline & $\pm \mathrm{SD}$ & 2.88 & 1.46 & 2.75 & 2.63 & \\
\hline \multirow{2}{*}{ Immediate bleaching } & Mean & $4.44^{\mathrm{c}}$ & $9.08^{\mathrm{ab}}$ & $9.73^{\mathrm{a}}$ & $5.69^{\mathrm{bc}}$ & \\
\hline & $\pm \mathrm{SD}$ & 1.45 & 1.08 & 3.44 & 1.34 & \\
\hline \multirow{2}{*}{ One Week } & Mean & $7.57^{\mathrm{b}}$ & $40.76^{\mathrm{a}}$ & $13.86^{\mathrm{b}}$ & $15.73^{\mathrm{b}}$ & \multirow{2}{*}{$<0.001^{*}$} \\
\hline & $\pm \mathrm{SD}$ & .51 & 12.10 & 1.65 & 2.96 & \\
\hline \multirow{2}{*}{ Fluoride } & Mean & $44.75^{\mathrm{b}}$ & $73.94^{\mathrm{a}}$ & $37.89^{b}$ & $21.30^{\mathrm{b}}$ & \multirow{2}{*}{$0.001^{*}$} \\
\hline & $\pm \mathrm{SD}$ & 4.18 & 29.12 & 11.13 & 3.56 & \\
\hline
\end{tabular}

Different superscripts in the same row are statistically different.

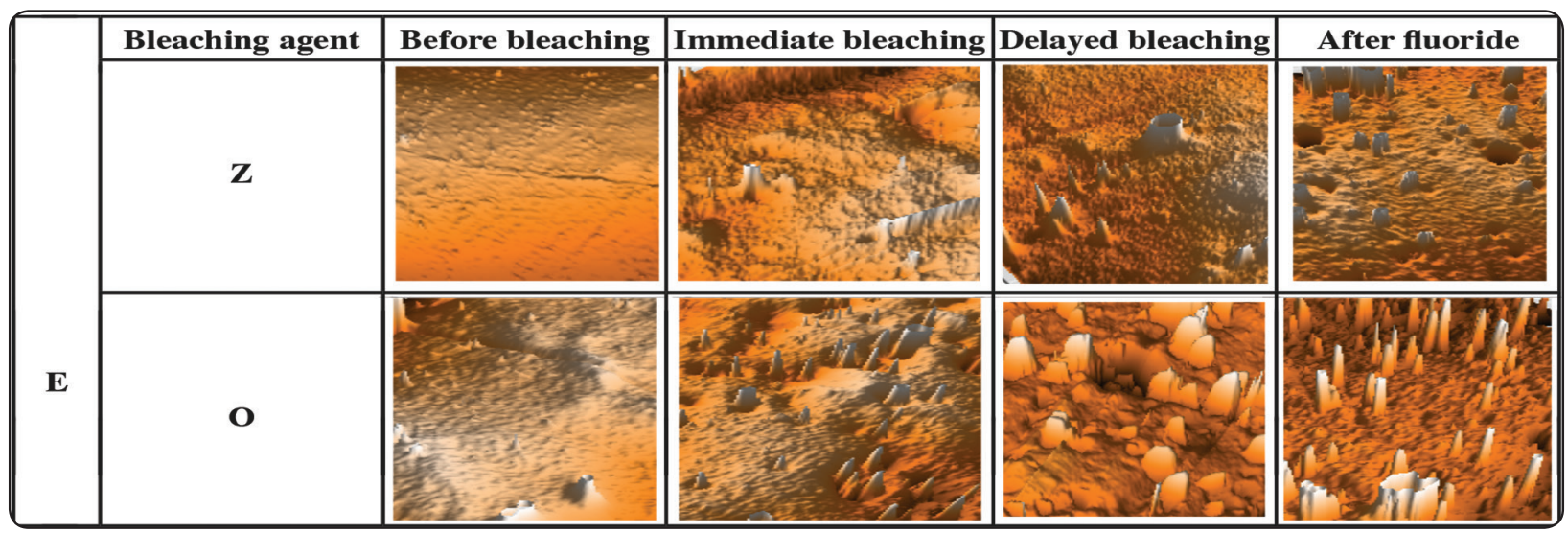

Fig. (1): 3D images of E.max CAD with different treatment. 


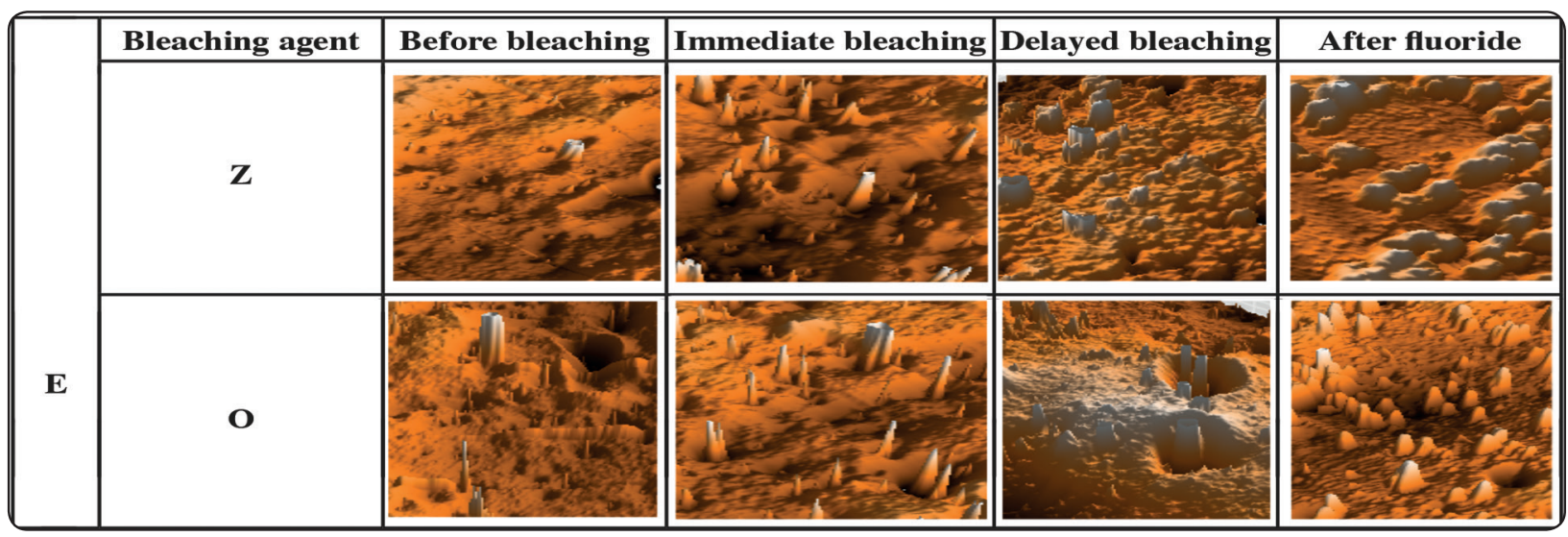

Fig. (2): 3D images of Vita Suprinity with different treatment.

\section{DISCUSSION}

\section{a) Discussion of the methodology:}

The continuous evolution in dentistry has allowed the remarkable success of all ceramic restorations, which in turn has encouraged manufacturer to develop new challenging materials supplied for CAD/CAM machines. ${ }^{4}$

Bleaching agents had reported adverse effects on different resin composite and hybrid ceramic materials $^{5,17-22}$, however there was a controversy about the effect of bleaching agent regarding the ceramic materials. ${ }^{6,23,24}$

At the end of the bleaching treatment, the presence of saliva and the use of fluorides are important to allow mineral saturation and increase the remineralization of tooth structure. Saliva could dilute the bleaching gel and reduce its concentration and the associated side effects on both tooth structure and restorative materials. To improve the uptake of fluoride and limit the amount of demineralization of the tooth, manufacturers have produced acidulated fluoride products. APF inhibits dental caries by approximately $30 \%$ to $40 \%$.

Color changes can be assessed either by visual and/or instrumental mechanisms. ${ }^{25}$ Instrumental techniques for example such as spectrophotometer eliminate the subjective interpretation of visual color comparison. CIE lab system was used in this study to detect minor color difference. $\Delta \mathrm{E}$ represents the numerical values between the 3 coordinates $\mathrm{L}^{*} \mathrm{a} \mathrm{b}^{*}$ and demonstrates the color change values. ${ }^{26,27}$ It was reported that ${ }^{28}$ if change in color value was $(\Delta \mathrm{E})$ $<1$, it was considered undetectable by human eye. But, if the change in color value was $1<(\Delta \mathrm{E})<3.3$, it was clinically acceptable and only skilled person can notice the changes. However, if color change was $\Delta \mathrm{E}>3.3$, it was observed easily and referred as not clinically acceptable. ${ }^{29,30}$

In addition, surface roughness is an important criterion of restorative materials. There are different techniques for surface analysis, ${ }^{31}$ AFM was used in this study for both quantitative and qualitative analysis of the ceramic surface because it provides one of the most accurate surface roughness measurement, flexible, and demonstrates more detailed images (nanometer scale) also, it produces 2D and 3D images which can be constructed at the same time.

\section{b) Discussion of the results:}

Regarding the effect of the bleaching agent used; Zoom $(\mathrm{Z})$ and Opalescence $(\mathrm{O})$ agents on color changes $\Delta \mathrm{E}$ of both tested ceramic specimens, the $\Delta \mathrm{E}$ values were increased in whitening effect in Vita Suprinity compared with that of the E.max CAD. This may be attributed to the higher translucency of Vita Suprinity compared to that of E.max as the 
color space values $\left(L^{*}, a^{*}, b^{*}\right)$ of Vita Suprinity ceramic before treatment were higher than those of E.max CAD. ${ }^{32}$

Regarding the E.max CAD and Vita Suprinity ceramic materials, the Opalescence agent showed higher statistically significant difference in mean $\Delta \mathrm{E}$ than that of the Zoom bleaching agent in all tested specimens of the ceramic materials. This may be attributed to the higher concentration of the active ingredients HP in the Opalescence agent. ${ }^{33}$

Comparing the four subgroups (EZ,EO,SZ\&SO) of treated ceramic materials, the highest color changes $\Delta \mathrm{E}$ occurred in Vita Suprinity treated with Opalescence agent (SO) for all tested conditions (I,D $\&$ F). This may be attributed to the fact of the higher color parameters $\left(\mathrm{L}^{*}, \mathrm{a}^{*} \& \mathrm{~b}^{*}\right)$ in Vita Suprinity before treatment than that of E.max CAD and the aggressive high concentration of Opalescence gel as it was applied on the specimens for 40 minutes. In addition, the extended effect of chemicallyactivated Opalescence agent leads to more color changes observed in all tested conditions. So, the effect of bleaching in the current study could be considered material dependent

Results of this study agreed with other studies ${ }^{34,35}$ although they used different ceramic materials and different concentrations of the bleaching agents. Results of this study disagreed with Zaki and Fahmy ${ }^{11}$ who reported no significant change in whitening after in-office bleaching agent and fluoride gel application in auto-glazed ceramic specimens. This may be related to the use of autoglazed ceramic where the firing cycle leads to sealing the milled ceramic surface without use of the external glaze.

Regarding the effect of fluoride application on the color of tested ceramic materials, there was a highly statistically significant difference in mean color change $(\Delta \mathrm{E})$ than bleaching application only. This could be explained by the presence of hydrofluoric acid in the APF gel which results in dissolution of the silica in the glass ceramic materials and forming a precipitate on the surface that affect color stability. These effects could have been enhanced using high concentrations of HP. The effect of fluoride application was higher in e.max CAD than that of the Vita Suprinity; this may be attributed to the composition of the Vita Suprinity as the presence of zirconia particles makes the surface more resistant to hydrofluoric acid effect than e.max that contains only the glass matrix and some oxides. These results agreed with Artopoulou et $\mathrm{al}^{36}$ and Pires-de-Souza et $\mathrm{al}^{37}$ although they used different types of ceramic materials. On the other hand, the results disagreed with Windeler et al, ${ }^{38}$ who reported the use of APF brushing gel on glass ceramics do not alter the ceramic and can be used without any negative effect on esthetic of ceramic used. This may be attributed to the less time of contact between APF and ceramic surface, different time of measurements, different bleaching agent concentrations and different measuring devices.

The hypothesis of this study was rejected as the in-office bleaching agents produced significant color changes on the restorative materials selected.

The results of this study revealed that both tested materials showed noticeable color changes after bleaching and the $\Delta \mathrm{E}$ values were greater than 3.3 that were considered clinically unacceptable based on previous reports ${ }^{28,29}$ except in the immediate measurements (EZI\&SZI). In those subgroups, the $\Delta \mathrm{E}$ values were greater than 2 that considered clinically acceptable but might be observed. This may be attributed to the mild effect of Zoom agent as it contains only $25 \% \mathrm{HP}$.

The degree of surface roughness seemed to be related to the composition and the microstructure of tested ceramic materials used. ${ }^{11}$ Comparing the results of the baseline with that of the immediate bleaching, baseline showed higher surface roughness $\mathrm{Ra}$. This may be attributed to the initial effect of the bleaching agent on the peaks present on the superficial surface of both ceramic materials leading to decrease in the distance between 
peaks and valleys resulting in decrease in surface roughness $(\mathrm{Ra})$ of tested ceramic materials.

Comparing the results of immediate bleaching (I) with delayed one (D), there was an increase in the $\mathrm{Ra}$ values in delayed bleaching $\mathrm{D}$ especially with Opalescence agent. This might be attributed to the high concentration of chemically-activated Opalescence which extends its effect even after removal of bleaching gels as some residual active elements may be entrapped into the valleys of the rough surface while in the Zoom bleaching agent, it is photo-activated so the effect of bleaching may be stopped after removal of the light source.

These results were in agreement with other studies ${ }^{6,18,35}$ although they used different ceramic materials with different bleaching concentrations. The results of this study were in disagreement with those of $12,39,40$, this may be related to the use of different types of ceramic materials, different bleaching concentration, and different measuring devices.

After fluoride treatment, the surface roughness increased more than after bleaching only in both tested ceramic materials. E.max CAD treated with Opalescence agent (EO) showed the highest $\mathrm{Ra}$ value changes. This may be attributed to the effect of APF combined with weak glass ceramic E.max and high concentration of Opalescence agent that extended its effect on surface dissolution leading to an exaggerated effect.

Results of this study were in agreement with those of Ural et $\mathrm{al}^{6}$, Mokhtar and McIntyre ${ }^{18}$ and Karakaya and Cengiz ${ }^{35}$ although there were no bleaching session before fluoride treatment. The hypothesis of this study was rejected, as use of bleaching systems and application of fluoride gel produced significant surface roughness changes on tested ceramic materials. It was reported that, if the mean surface roughness change was $>200$ $\mathrm{nm}$, it was referred as not clinically acceptable and produced plaque accumulation with higher risk of caries and periodontal diseases. This value was used in this study to evaluate the clinical effect of amount of surface roughness changes. Accordingly, the results of this study showed that bleaching and/or fluoride application resulted in clinically acceptable surface roughness changes (Ra values) as they were less than $200 \mathrm{~nm}$.

The results collected in this study proved that, there was a statistically significant positive (parallel) correlation between color parameters and roughness values $(\mathrm{Ra})$. The high reported color change $(\Delta \mathrm{E})$ values following the applied treatments might also be attributed to the reported increase in the mean surface roughness $(\mathrm{Ra})$ in all tested ceramics; this is because the color of an object depends on its surface spectral reflectance. These results agreed with Motro and Kursoglu, ${ }^{41}$ who reported that the staining of ceramic is directly related to surface roughness changes which occur after different surface treatments.

\section{CONCLUSIONS}

Under the limitation of this study, it is concluded that:

1. The influence of in-office bleaching agents on color was material dependent.

2. The immediate in-office bleaching agents produced acceptable clinical effect on the color of tested ceramic materials.

3. The delayed in-office bleaching and fluoride application produced unacceptable clinical effect on color of ceramic materials.

4. The changes in color and surface roughness were time dependent.

5. The in-office bleaching and fluoride gel application resulted in clinically acceptable surface roughness changes.

6. Fluoride application following bleaching of the ceramic materials showed higher color and surface roughness changes than bleaching application only in all tested groups. 


\section{RECOMMENDATIONS}

Under the parameters of this study; the following recommendations could be derived:

1. The clinician should protect the ceramic restoration before bleaching sessions and/or fluoride application to avoid esthetic deteriorations.

2. It would be useful to support the results of this study with the studies that aimed to assess the effect of fluoride application immediately after bleaching sessions on ceramic materials and others that assess the effect of different remineralizing agents after bleaching of ceramic materials

3. The effect of different concentrations and application times could be further assessed to give better recommendations for the best protocols of bleaching and fluoride agents application that ensures optimum color stability and surface topography characteristics.

\section{REFERENCES}

1. Rekow ED, Silva NRFA, Coelho PG, Zhang Y, Guess P, Thompson VP. Performance of dental ceramics: challenges for improvements. J Dent Res. 2011;90(8):937-952.

2. Chen Y M, Smales RJ, Yip KH-K, Sung WJ. Translucency and biaxial flexural strength of four ceramic core materials. Dent Mater. 2008;24(11):1506-1511.

3. Bahgat SFA, Basheer RR, El Sayed SM. Effect of zirconia addition to lithium disilicate ceramic on translucency and bond strength using different adhesive strategies. Dent $\mathrm{J}$. 2015;61(5):4519-4533.

4. Edward A. McLaren MDC; and Johan Figueira D. Updating classifications of ceramic dental materials: A guide to material selection. ADA CERP. 2015;36(6):739-744.

5. Yazıcı AR, Atalay C, Bektaş B. The effect of laser-assisted in-office bleaching on surface roughness of different types of restorative resins. Clin Dent Res. 2016;40(1):3-10.

6. Ural Ç, Gençer Y, Tarakçi M, Aslan Ma, Arici S, Tatar N. Effect of bleaching agents on surface texture of feldspathic ceramic. J Exp Clin Med. 2014;31(3):177-181.

7. Wattanapayungkul P, Yap AUJ, Chooi KW, Lee MFLA, Selamat RS, Zhou RD. The effect of home bleaching agents on the surface roughness of tooth-colored restoratives with time. Oper Dent. 2004;29(4):398-403.

8. Sarafianou A, Kamposiora P, Papavasiliou G, Goula H. Matching repeatability and interdevice agreement of 2 intraoral spectrophotometers. J Prosthet Dent. 2012;107(3):178-185.

9. Lasserre J-F, Pop-Ciutrila I-S, Colosi H-A. A comparison between a new visual method of colour matching by intraoral camera and conventional visual and spectrometric methods. J Dent. 2011;39:e29-e36.

10. Scheibe KGBA, Almeida KGB, Medeiros IS, Costa JF, Alves CMC. Effect of different polishing systems on the surface roughness of microhybrid composites. J Appl Oral Sci. 2009;17(1):21-26.

11. Zaki AA, Fahmy NZ. The effect of a bleaching system on properties related to different ceramic surface textures. J Prosthodont. 2009;18(3):223-229.

12. Ourique SAM, Arrais CAG, Cassoni A, Ota-Tsuzuki C, Rodrigues JA. Effects of different concentrations of carbamide peroxide and bleaching periods on the roughness of dental ceramics. Braz Oral Res. 2011;25(5):453-458.

13. Armênio R V, Fitarelli F, Armênio MF, Demarco FF, Reis A, Loguercio AD. The effect of fluoride gel use on bleaching sensitivity: a double-blind randomized controlled clinical trial. J Am Dent Assoc. 2008;139(5):592-597.

14. Bizhang M, Seemann R, Duve G, et al. Demineralization effects of 2 bleaching procedures on enamel surfaces with and without post-treatment fluoride application. Oper Dent. 2006;31(6):705-709.

15. Nascimento WC, Gomes Y do SB de L, Alexandrino LD, Costi HT, Silva JOC, Silva CM. Influence of fluoride concentration and $\mathrm{pH}$ Value of $35 \%$ hydrogen peroxide on the hardness, roughness and morphology of bovine enamel. J Contemp Dent Pract. 2014;15(4):392-398.

16. Butler CJ, Masri R, Driscoll CF, Thompson GA, Runyan DA, Anthony von Fraunhofer J. Effect of fluoride and $10 \%$ carbamide peroxide on the surface roughness of lowfusing and ultra low-fusing porcelain. J Prosthet Dent. 2004;92(2):179-183.

17. Luque-Martinez I, Reis A, Schroeder M, et al. Comparison of efficacy of tray-delivered carbamide and hydrogen peroxide for at-home bleaching: a systematic review and meta-analysis. Clin Oral Investig. 2016;20(7):1419-1433.

18. Mokhtar K, McIntyre J. Analysis of etching of toothcoloured restoratives by different acidulating systems in topical fluoride gels. J Phys Sci. 2012;23(1):15-28. 
19. Al-Ameedee AH, Ragab HM, Osman E, Salameh Z. Evaluation effect of an in-office zoom bleaching gel agent on the surface texture of three contemporary restorative materials. Tanta Dent J. 2015;12(3):168-177.

20. Yildiz E, Karaarslan ES, Simsek M, Ozsevik AS, Usumez A. Color stability and surface roughness of polished anterior restorative materials. Dent Mater J. 2015;34(5):629-639.

21. Zavanelli AC, Mazaro VQ, Silva CR, Zavanelli RA, Mancuso DN. Surface roughness analysis of four restorative materials exposed to $10 \%$ and $15 \%$ carbamide peroxide. Int J Prosthodont. 2011;24(2):155-157.

22. Taher NM. The effect of bleaching agents on the surface hardness of tooth colored restorative materials. J Contemp Dent Pract. 2005;6(2):18-26.

23. Ccahuana VZS, Özcan M, Mesquita AMM, Nishioka RS, Kimpara ET, Bottino MA. Surface degradation of glass ceramics after exposure to acidulated phosphate fluoride. J Appl Oral Sci. 2010;18(2):155-165.

24. Kamala KKR, Annapurni H. Evaluation of surface roughness of glazed and polished ceramic surface on exposure to fluoride gel, bleaching agent and aerated drink: An in vitro study. J Indian Prosthodont Soc. 2006;6(3):128.

25. Llena C, Lozano E, Amengual J, Forner L. Reliability of two color selection devices in matching and measuring tooth color. J Contemp Dent Pract. 2011;12(1):19-23.

26. Derdilopoulou F V, Zantner C, Neumann K, Kielbassa AM. Evaluation of visual and spectrophotometric shade analyses: a clinical comparison of 3758 teeth. Int J Prosthodont. 2007;20(4):414-416.

27. Gómez-Polo C, Gómez-Polo M, Celemin-Viñuela A, Martínez Vázquez De Parga JA. Differences between the human eye and the spectrophotometer in the shade matching of tooth colour. J Dent. 2014;42(6):742-745.

28. Stawarczyk B, Sener B, Trottmann A, Roos M, Mmerle Chf. Discoloration of manually fabricated resins and industrially fabricated CAD/CAM blocks versus glass-ceramic: Effect of storage media, duration, and subsequent polishing. Dent Mater J. 2012;31(3):377-383.

29. Borges ALS, Costa AKF, Saavedra GSF a, Komori PCP, Borges AB, Rode SM. Color stability of composites: effect of immersion media. Acta odontológica Latinoam. 2011;24(2):9-193.
30. De Alencar e Silva Leite ML, Da Cunha Medeiros e Silva FDS, Meireles SS, Duarte RM, Maciel Andrade AK. The effect of drinks on color stability and surface roughness of nanocomposites. Eur J Dent. 2014;8(3):330-336.

31. Kukiattrakoon B. Effect of Acidic Agents on Surface Roughness of Dental Ceramics. Dent Res J. 2011;8(1):6-15.

32. Bahgat SFA, Basheer RR, M Ess. Effect of zirconia addition to lithium disilicate ceramic on translucency and bond strength using different adhesive strategies. Egypt Dent J. 2015;61:4519-4533.

33. Perdigão J. Tooth Whitening: An Evidence-Based Perspective.; 2016.

34. Kara HB, Aykent F, Ozturk A. The effect of bleaching agents on the color stability of Ceromer and porcelain restorative materials in-Vitro. Oper Dent. 2013;38(1):1-8.

35. Karakaya İ, Cengiz E. Effect of 2 Bleaching Agents with a Content of High Concentrated Hydrogen Peroxide on Stained 2 CAD/CAM Blocks and a Nanohybrid Composite Resin: An AFM Evaluation. Biomed Res Int. 2017;2017(6347145):1-11.

36. Artopoulou II, Powers JM, Chambers MS. In vitro staining effects of stannous fluoride and sodium fluoride on ceramic material. J Prosthet Dent. 2010;103(3):163-169.

37. Pires-de-Souza FC, Contente MM, Alandia-Román CC, Vicente SA TR. Effect of bleaching agent and topical fluoride application on color and gloss of dental ceramics. Gen Dent. 2016;64(6):16-20.

38. Windeler T. Amine fluoride does not cause disintegration of glass ceramics. Schweizer Monatsschrift fur Zahnmedizin. 2004;114(1):41-47.

39. Federal U, Tobias R, Neto M. Evaluation of the effects of $15 \%$ carbamide peroxide and $38 \%$ hydrogen peroxide on the ceramic surface IPS inline. Cerâmica. 2015;24(2): 153-161.

40. Ferreira H de A, Carlo HL, e Silva FDCM. Influence of bleaching agents on surface properties (roughness and microhardness) of dental ceramics. Cerâmica. 2016; 62(361):55-59.

41. Motro PFK, Kursoglu P. Effects of different surface treatments on stainability of ceramics. J Prosthet Dent. 2012; 108(4):231-237. 